\title{
Topological optimization of patterned silicon anode by finite element analysis
}

\author{
Shanghong DUAN ${ }^{1}$, Alexander M. LAPTEV ${ }^{1 *}$, Robert MÜCKE ${ }^{1}$, Dmitri L. DANILOV ${ }^{2,3}$, \\ Peter H. L. NOTTEN ${ }^{2,3}$, Olivier GUILLON ${ }^{1,4}$
}

\author{
${ }^{1}$ Institute of Energy and Climate Research, Materials Synthesis and Processing (IEK-1), Forschungszentrum Jülich GmbH, 52425 Jülich, Germany \\ ${ }^{2}$ Institute of Energy and Climate Research, Fundamental Electrochemistry (IEK-9), Forschungszentrum Jülich GmbH, 52425 Jülich, Germany \\ ${ }^{3}$ Department of Electrical Engineering, Eindhoven University of Technology, $5600 \mathrm{MB}$ Eindhoven, The Netherland \\ ${ }^{4}$ Jülich-Aachen Research Alliance: JARA-Energy, 52425 Jülich, Germany \\ *Corresponding author: laptev@gmx.net \\ Tel.:+49-2461-61-2248; fax: +49-2461-61-8210
}

\begin{abstract}
A silicon-based anode in lithium-ion battery exhibits several times higher gravimetric energy storage capacity compared to an established carbon-based anode. However, the cycling performance of the silicon anode is poor due to the extremely large volume variation during the intercalation of lithium ions. The micro-structuring of silicon facilitates cycling performance. In particular, patterned microstructures are discussed as a possible solution. The large volumetric change can be adopted in such structures by bending walls and rotation around fixed vertexes. Nevertheless, the cycling performance of known patterned anodes remains poor due to plastic deformations. In this paper, a new squarebased-patterned silicon anode is proposed and analyzed using the finite element method. The maximal stress in the topologically optimized structure is below the yield strength of lithiated silicon. In contrast to known structures, the deformed pattern of the new structure is explicitly defined by its initial geometry. A similar modification of the honeycomb-based-patterned anode leads to a slightly larger bending stress, but still below the yield stress of lithiated silicon. The related pure elastic deformation behavior is favorable to a prolonged cycling life of the microstructured silicon anode. The developed approach can be applied for analysis of other severely swelling metamaterials.
\end{abstract}

Keywords: patterned silicon anode, metamaterial, volumetric expansion, chemo-mechanics, finite element analysis, topological optimization

\section{Introduction}

\subsection{Silicon anode}

The application in Li-ion batteries is continuously growing ranging from micro-sized energy sources in sensors and medical devices to large battery packs for automotive and residential applications. The core of battery is the electrochemical cell where processes leading to charge and discharge of battery occur. In general, the cell consists of two electrodes and electrolyte. During charge, lithium ions move from the positive electrode (cathode) to the negative electrode (anode) through the electrolyte. In the course of discharge, they move in opposite direction. In accordance with this motion, lithium ions are inserted into lattice of anode (lithiation) or extracted from its lattice (delithiation). This process (called the intercalation) results in reversible expansion and contraction of anode material during charge and discharge. This behavior is similar in appearance to thermal expansion, but the volume change is much larger. In particular, the relative volumetric expansion of established graphite anode is about $10 \%$. Even larger volumetric variation shows anode manufactured from silicon ( $\mathrm{Si}$ anode). This anode is discussed as a possible alternative to graphite anode due to its tenfold higher gravimetric storage capacity [1]. A fully charged $\mathrm{Si}$ anode exhibits a relative increase in volume of about $380 \%$ [2]. Such large and reversible volumetric variation is unique for solids. The enormous change in volume of Si anode can result in large stresses due to gradient in lithium concentration or if anode is constrained. Therefore, it is not surprising that the fragmentation and poor cycling performance of silicon anode are frequently observed [3]. A gradient in lithium concentration can be minimized by application of low charge (and discharge) rate. However, this is not desirable in practical application of batteries. An alternative to slow charge could be the use of silicon anodes in the form of micro or nano-sized powders, wires, pillars or thin films [4].

\subsection{Chemo-mechanical analysis}

Discussion of electrochemical performance of various $\mathrm{Si}$ anodes is beyond the scope of this paper. We only want to emphasize that such anodes are new, interesting and relevant for practice objects for mechanical analysis. This analysis requires a special approach which is referred to as the chemomechanics. In general, chemo-mechanics deals with a coupled analysis of mechanical and chemical problems. The intercalation of Li ions in silicon matrix is usually discussed as a diffusional process. Thus, during chemo-mechanical analysis of silicon anode a diffusional-structural problem must be solved. The examples of chemo-mechanical analysis of microsized Si-anodes are widely presented in the literature. Only several representative works are mentioned below. Wang et al. analyzed the chemo-mechanical behavior of silicon nanopowders [5, 6]. Yang et al. discussed deformation and fracture strains of silicon nanowires [7]. Phar et al. modeled the mechanical response of silicon nano-sized pillars on lithium intercalation [8]. Wang et al. and Gwak et al. studied mechanics of lithiated $\mathrm{Si}$ anodes in the form of thin films [5,9]. In general, two main challenges in chemo-mechanical analysis must be addressed. First, the mechanical properties of micro-sized silicon must be determined. This is a complex task, because of small size of object to be investigated. Moreover, the properties must be defined at different lithium concentrations. At present, the elastic properties of lithiated silicon are satisfactory 
investigated $[10,11]$. The non-elastic properties are still under investigation by sophisticated experiments [12] or by firstprinciples, density function and molecular dynamics modeling $[13,14]$. Second, the dependence of mechanical properties on lithium concentration must be implemented in a FEM model and related code, which are usually used for chemo-mechanical analysis. For instance, the widely used FEM package ANSYS provides solution of coupled diffusional-structural problems. At the same time, direct relation of properties with solute concentration is not available so far.

\subsection{Honeycomb-and square-patterned silicon anode}

Recently, Baggetto et al. have developed the honeycombpatterned micro-structured $\mathrm{Si}$ anode and produced it by photolithography and etching [15]. An example of such an anode in lithiated state is shown in Fig. 1a. Baggetto et al. observed the position of vertices in honeycomb structure remaining fixed during entire lithiation/delithiation cycle. Thus, during $\mathrm{Li}$ intercalation and swelling/contraction the honeycomb structure is internally self-constrained. As a result, the corresponding patterned anode did not change its dimension in plane despite the large volumetric variation of basic material (e.g. silicon). Taking into account the size and the number of primary cells, the honeycomb-patterned microstructure can be classified as a metamaterial. This novel type of structures is widely discussed in the modern literature on mechanics of materials [16]. At the same time, their mechanical analysis is limited to cases of an external loading. Bhandakkar and Johnson were the first to investigate the mechanical behavior of severely swelling honeycomb-shaped $\mathrm{Si}$ anodes by FEM and ABAQUS code [17]. However, the dependence of mechanical properties on lithium concentration was not considered in this work. Laptev et al. included this dependence during FEM modeling by ANSYS [18]. The appearance of plastic deformation during lithiation and bending of self-constrained honeycomb walls was predicted. The accumulation of plastic strain during charge/discharge cycling can lead to fatigue and fracture of the honeycomb walls. The failure of honeycomb microstructures after several cycles was also observed in experiments (Fig. 1b).

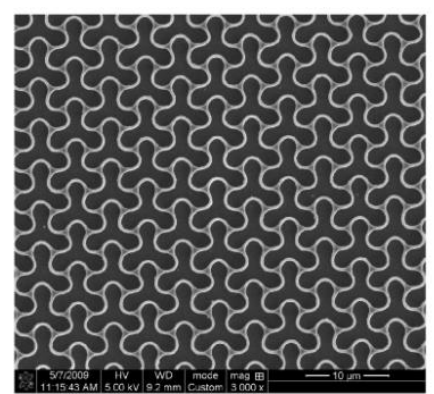

(a)

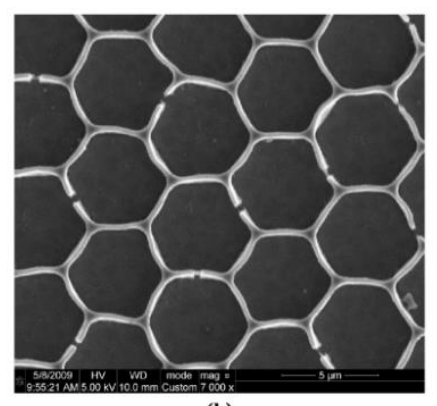

(b)
Fig. 1. Honeycomb-patterned Si anode [15]: (a) after lithiation, (b) fractured honeycomb walls.

Further optimization of honeycomb geometry has shown that the plastic deformation can be decreased or even eliminated, but only at the expense of a drastic reduction in footprint-area-specific storage capacity [18]. Subsequent analysis of the square-patterned microstructure storing the same energy per unit footprint-area has shown a lower - but still high - bending stress. Therefore, other patterned structures were considered in the present work.

\subsection{Proposed structure}

The idea was to mitigate the lithiation-induced distortion through the rotation of structural elements around vertices while minimizing their bending. In line with this consideration, a new square-based structure with a unit cell schematically shown as ADEF in Fig. 2 was developed. Due to the periodic symmetry of such a structure, the position of vertices A, D, E and $\mathrm{F}$ remains fixed during entire lithiation/delithiation cycle. The segments $\mathrm{AB}, \mathrm{DC}, \mathrm{EG}$ and $\mathrm{FH}$ rotate around vertices, thus minimizing the bending of segments $\mathrm{BC}$ and $\mathrm{GH}$ as well as the related strain and stress.

Upon further analysis and discussion of this structure, Baggetto's basic honeycomb structure was taken as a reference [15]. In particular, a maximal lithiation of $80 \%$ theoretical value was assumed, in accordance with the highest level achieved in experiments $[15,18]$. An initial wall thickness of $t_{0}=0.25 \mu \mathrm{m}$ and an initial wall height of $h_{0}=1.1 \mu \mathrm{m}$ were taken as in the basic honeycomb structure. The initial length of segments $\mathrm{BC}$ and $\mathrm{GH}$ (later denoted as $L_{0}$ ) was varied from $6.0 \mu \mathrm{m}$ to $8.0 \mu \mathrm{m}$ and the initial length of segments $\mathrm{AB}=\mathrm{DC}=\mathrm{EG}=\mathrm{FH}\left(\right.$ denoted as $\left.R_{0}\right)$ was varied from $1.0 \mu \mathrm{m}$ to $2.0 \mu \mathrm{m}$. The fillet radius between the walls was taken as $r_{0}=0.1 \mu \mathrm{m}$. The footprint-area-specific storage capacity $S_{f a}$ was normalized by a value of $S_{f a b h}=88.65 \mu \mathrm{Ah} \cdot \mathrm{cm}^{-2}$, which is the capacity of Baggetto's honeycomb [18]. The aim of our study was to develop an approach for finding out a combination of $R_{0}$ and $L_{0}$ resulting in pure elastic deformation after lithiation and providing a footprint-area-specific storage capacity no less than that of the basic honeycomb structure. The structural analysis was performed using the finite element modeling. The geometry of cell was optimized by the linear programming method.

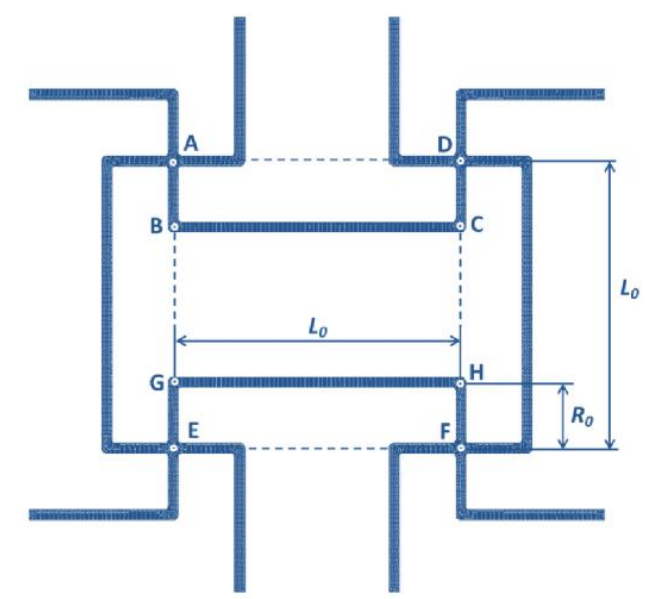

Fig. 2. Representative unit cell of proposed square-based structure.

\section{Modeling procedure}

\subsection{Theoretical background}

The isotropic chemo-mechanical behavior of lithiated silicon was assumed. The initially amorphous silicon (a-Si) was considered. The retention of amorphous sate during lithium intercalation was assumed. Lithiation was modeled as diffusion flow of $\mathrm{Li}$ ions (and related electric charges) into $\mathrm{Si}$ matrix 
through the free surface of a representative unit cell. This process can be described by Fick's laws (1) and (2) with a diffusion coefficient of $D=5.1 \cdot 10^{-4} \mu \mathrm{m}^{2} \cdot \mathrm{s}^{-1}$ taken from the experimental work of Ding et al. [19]

$$
\begin{gathered}
\boldsymbol{j}=-D \cdot \nabla\left(C_{\max } \cdot \bar{c}\right) \\
\partial\left(C_{\max } \cdot \bar{c}\right) / \partial t=D \cdot \nabla^{2}\left(C_{\text {max }} \cdot \bar{c}\right)
\end{gathered}
$$

$\boldsymbol{j}$ is the lithium flux vector (or the current density vector), $t$ is the time, and $C_{\max }$ is the maximal volumetric lithium concentration (or volumetric storage capacity), $\bar{c}$ is the normalized Li concentration (or normalized storage capacity) defined as

$$
\bar{c}=C / C_{\max }
$$

$C$ is the volumetric lithium concentration (or stored charge) and $\bar{c} \in[0,1]$. The value of $C_{\max }$ is not a constant, because of volume change during lithiation and delithiation. The corresponding dependence is described by the formula

$$
C_{\text {max }}=C_{V} \cdot\left(V / V_{0}\right)^{-1}=C_{V} \cdot \exp (-3 \cdot \beta \cdot \bar{c})
$$

$V$ is the volume of lithiated silicon, $V_{0}$ is the volume of silicon before lithiation, $\beta$ is the chemical expansion coefficient explained below, $C_{V}$ is the volumetric storage capacity of fully lithiated silicon. $C_{V}$ is determined as $C_{V}=C_{g} \cdot \rho_{S i}$. Here $C_{g}$ and $\rho_{S i}$ are the gravimetric storage capacity and the density of silicon. A value of $C_{V}=8303 \mathrm{~mA} \cdot \mathrm{h} \cdot \mathrm{mm}^{-3}$ was used in our calculation in accordance with the data provided in the paper [20]. A footprint area related charging rate of $j_{\mathrm{ch}}=75 \mu \mathrm{A} \cdot \mathrm{cm}^{-2}$ was taken from the experimental work of Baggetto et al. [15]. With this charging rate the basic honeycomb can be fully charged in $t_{\mathrm{ch}}=S_{f a b h} / j_{\mathrm{ch}}=1.18 \mathrm{~h}$. Such a charging rate corresponds to a Crate of $t_{\mathrm{ch}}{ }^{-1}=0.846 \mathrm{~h}^{-1}$, which is the established measure of charge rate in electrochemistry. A charge of $80 \%$ can be achieved after $0.944 \mathrm{~h}$ charging.

An incremental approach was used during FEM modeling. The total strain increment was considered as a sum of intercalation-induced and elastic strain increments, i.e.

$$
d e_{i j}=d e_{i j}^{L i}+d e_{i j}^{e}
$$

The linear dependence of lithiation-induced increment of Hencky strain on the increment of $\bar{c}$ was applied according to Pursin [21] and Pharr et al. [22]

$$
d e_{i j}^{L i}=\beta \cdot d \bar{c} \cdot \delta_{i j}
$$

The chemical expansion coefficient was taken as $\beta=0.445$ from our previous work [18]. Since our goal was to avoid plastic deformation, the perfectly elastic behavior of lithiated silicon was considered. The increments of elastic strain were determined from Hooke's law

$$
d e_{i j}^{e}=E^{-1} \cdot\left(d \sigma_{i j}-v \cdot\left(d \sigma_{k k} \cdot \delta_{i j}-d \sigma_{i j}\right)\right)
$$

The experiments revealed that Young's modulus $E$ and Poisson ratio $v$ are varied with $\mathrm{Li}$ content. Based on Hertzber's et al. results for thin films, the linear relationship between Young's modulus and normalized Li concentration was used

$$
E=E_{0}-E_{0} \cdot\left(1-E_{1}\right) \cdot \bar{c}
$$

where $E_{0}=90 \mathrm{GPa}$ is the Young's modulus of pristine silicon, $E_{1}=12 \mathrm{GPa}$ is the Young's modulus of fully lithiated silicon [10]. The linear dependence between Poisson ratio $v$ end $\mathrm{Li}$ concentration was adopted from the first-principles study of lithiation by Shenoy et al. [11]

$$
v=v_{0}-v_{0} \cdot\left(1-v_{1}\right) \cdot \bar{c}
$$

where $v_{0}=0.28$ is the Poisson ratio of original silicon, $v_{1}=0.24$ is the Poisson ratio of fully lithiated silicon.

\subsection{FEM implementation}

\subsection{1. $2 D$ model}

The mathematical model was realized by ANSYS 15.0 finite element software (ANSYS Inc., Canonsburg, USA) as an APDL code. The deformation of a single cell representing the entire structure was modeled. The representative cell was considered as a 2D object under in-plain loading because the height does not influence significantly the stress state of the structure. Symmetric and periodic boundary conditions were applied. The microstructure was assumed to be in mechanical and electrical sliding contact with the underlying substrate. Frictionless displacement was assumed. The finite element PLANE 223 with structural-thermal-diffusional analysis capability and elements with a size of around $0.05 \mu \mathrm{m}$ were used. Free meshing was applied. Accuracy in determining of large deformations was ensured by the incremental approach and by the use of Hencky strain. The maximal volumetric storage capacity of silicon was transformed to the in-plain storage capacity $C_{2 D}$, taking into account the geometry of the discussed structure, i.e. $C_{2 D}=C_{V} \cdot h_{0}$. Then, by analogy with (4), the maximal in-plain storage capacity was defined by the formula

$$
C_{\text {max }}^{2 D}=C_{2 D} \cdot\left(V / V_{0}\right)^{-2 / 3}=C_{2 D}(-2 \cdot \beta \cdot \bar{c})
$$

To apply the load the total current flowing into the unit cell was first calculated by $J=j_{c h} \cdot\left(L_{0}\right)^{2}$. Then, the total normalized concentration flux was determined by division of $J$ by the maximal in-plane storage capacity at normalized concentration equal to one

$$
j=J / C_{2 D} \cdot(2 \cdot \beta)
$$

The value of the concentration flux per one node of the representative cell was computed. The obtained value was applied to all nodes in the FE model.

\subsubsection{Use of thermal analogy}

As was mentioned above, the ANSYS software does not support directly the dependence of mechanical properties on solute concentration. The simplest method to overcome this problem is the use of analogy between diffusional and thermal analysis. This approach is frequently used in the literature for study of diffusional processes [7, 8, 21-23]. The method is based on formal similarity of Fick's equations for diffusion 
analysis (1), (2) and Fourier equation (12) and heat equation (13) in thermal analysis

$$
\begin{gathered}
\boldsymbol{q}=-k \cdot \boldsymbol{\nabla} T \\
\partial T / \partial t=\gamma \cdot \nabla^{2} T
\end{gathered}
$$

where $\boldsymbol{q}$ is the heat flux vector, $k$ is the thermal conductivity and $T$ is the temperature. The thermal diffusivity $\gamma$ is related to the specific heat capacity $C_{p}$ and the density $\rho$ by the formula

$$
\gamma=k /\left(C_{p} \cdot \rho\right)
$$

In our analysis the thermal conductivity $k$ was assumed to be numerically equal to the diffusivity $D$ i.e. $\mathrm{k}=5.1 \cdot 10^{-}$ ${ }^{4} \mathrm{~W} \cdot \mu \mathrm{m}^{-1} \cdot \mathrm{C}^{-1}$. A value of $C_{p}$ was taken equal to $C_{\max }^{2 D}$ in dependence on normalized concentration. A value of $1 \mathrm{~g} \cdot \mu \mathrm{m}^{-3}$ was taken for density. The normalized concentration flux $\boldsymbol{j}$ was interpreted as the thermal flux $\boldsymbol{q}$. The temperature resulted in thermal analysis was considered as the normalized lithium concentration. If at any step of calculation the temperature exceeded $1{ }^{\circ} \mathrm{C}$, a value of $\mathrm{T}=1^{\circ} \mathrm{C}$ was applied. Thus the distribution of lithium concentration was determined. Then, calculation of lithiation induced strain increments was done owing to similarity of equation (6) for lithiation induced strain and equation (15) for strain induced by thermal expansion

$$
d e_{i j}^{T}=\alpha \cdot d T \cdot \delta_{i j}
$$

where $\alpha$ is the thermal expansion coefficient (TEC). In our analysis the TEC was taken numerically equal to the chemical expansion coefficient, i.e. $\alpha=0.445 \mathrm{C}^{\circ}-1$. The resulting strain increments were interpreted as the lithium induced one. The ANSYS code directly incorporates the dependence of elastic moduli on temperature. Thus, the stress increments were calculated using Hooke's law (7) with the moduli depending on lithium concentration in accordance with the equations (8) and (9). Prescribed level of lithiation was simulated through the variation in charging time from 0 to $0.944 \mathrm{~h}$, i.e. from 0 to 0.8 averaged concentration.

\subsection{Optimization approach}

As evident from Fig. 2, the proposed structure has two independent geometrical parameters: $R_{0}$ and $L_{0}$. These parameters determine not only the footprint-area-specific storage capacity, but also deformation of the entire structure. An increase in $R_{0}$ leads to an enlargement of segments $\mathrm{AB}, \mathrm{DC}$, $\mathrm{EG}, \mathrm{FH}$ after rotation caused by lithiation and swelling. As a result, the bending of segments $\mathrm{BC}, \mathrm{GH}$ and associated bending stress are decreased. Another effect of the increase in $R_{0}$ is the growth of footprint area, but the maximal charge can be limited by the earlier touch of opposing walls. An increase in $L_{0}$ leads to a reduction in wall deflection and therefore bending stresses but also results in a reduction of footprint-area-specific storage capacity. Therefore, the combination of $R_{0}$ and $L_{0}$ parameters must be optimized based on certain criteria. In the present study, we decided that after full charging, the maximal equivalent (von Mises) stress must be below the yield stress and that the relative storage capacity $r_{s c}=S_{f a} / S_{f a b h}$ must be no less than one $\left(r_{\mathrm{sc}} \geq 1\right)$. In addition, the overlap of opposing walls must be excluded.
The relative storage capacity of the proposed structure was determined from the initial geometry of the unit cell. The volume of silicon in such a cell was estimated using $V_{s i}=2 \cdot\left(L_{0}+2 \cdot R_{0}\right) \cdot t_{0} \cdot h_{0}$. The footprint area is equal to $A_{f a}=L_{0}^{2}$. Thus, the storage capacity per unit footprint area is $S_{f a}=V_{s i} / A_{f a}=2 \cdot\left(1+2 \cdot R_{0} / L_{0}\right) \cdot t_{0} \cdot h_{0} / L_{0} \cdot C_{V}$, where $C_{V}$ the volumetric storage capacity of $\mathrm{Si}$ (see section 2.1 ). Thus, the final formula for relative storage capacity is $r_{s c}=20.6 \cdot\left(1+2 \cdot R_{0} / L_{0}\right) \cdot t_{0} / L_{0}$. The optimization of proposed structure was realized using the linear programming method. Over the course of optimization, 121 FEM calculations were performed according to a matrix of $11 \times 11$ data points $\left(R_{0} \times L_{0}\right)$. The value of $R_{0}$ was varied from $1.0 \mu \mathrm{m}$ to $2.0 \mu \mathrm{m}$ with an increment of $0.1 \mu \mathrm{m}$, whilst the value of $L_{0}$ was varied from $6.0 \mu \mathrm{m}$ to $8.0 \mu \mathrm{m}$ with an increment of $0.2 \mu \mathrm{m}$.

\section{Results and discussion}

\subsection{Square-based microstructure}

An example of simulation result is shown in Fig. 3. The lithium distribution was slightly inhomogeneous with a higher concentration in the walls and a lower concentration in the vicinity of vertices. The mainly homogeneous lithiation of the walls was found. The stress distribution across the walls is typical for bending with tension, compression regions, and the neutral line. The maximal equivalent stress $\left(\sigma_{\text {eq.max }}\right)$ occurs at the top (tension region) and bottom (compression region) points in the cross section of the deformed wall.

The results of optimization analysis are shown in Fig. 4. The graph is divided into four regions. The region I corresponds to a combination of $R_{0}$ and $L_{0}$ parameters, leading to the contact of opposing walls before $80 \%$ lithiation. The region II relates to the combination of $R_{0}$ and $L_{0}$ resulting in too low storage capacity $\left(r_{s c}<1.0\right)$. In the region III, the equivalent stress is larger than the yield stress $(0.72 \mathrm{MPa})$ and plastic deformation occurs. All applied criteria are fulfilled in the feasible region IV. In this region, the lowest value of $\sigma_{\text {eq. } \max }=0.58 \mathrm{GPa}$ relates to the combination of $R_{0}=1.52 \mu \mathrm{m}$ and $L_{0}=7.4 \mu \mathrm{m}$ parameters as denoted by the asterisk. This value of $\sigma_{\text {eq.max }}$ is noticeably less than the maximal equivalent stress in the basic honeycomb and in basic square-patterned microstructures described earlier [18]. We consider the corresponding $R_{0}-L_{0}$ combination and associated geometry to be optimized. It is worth noticing that with optimized geometry, the opposing walls come in contact with each other at about $80 \%$ lithiation. The data for the optimized square-based structure are also presented in Table 1, contrasting with similar data for the basic honeycomb structure and for the optimized new honeycombbased structure. The new honeycomb-based structure is discussed in the section 3.3.

Table 1. Optimized geometry and related equivalent stress after $80 \%$ lithiation

\begin{tabular}{lccccc}
\hline Structure & $\begin{array}{l}R_{0}, \\
\mu \mathrm{m}\end{array}$ & $\frac{R_{0}}{t_{0}}$ & $\begin{array}{l}L_{0}, \\
\mu \mathrm{m}\end{array}$ & $\frac{L_{0}}{t_{0}}$ & $\begin{array}{c}\sigma_{\text {eq.max }}, \\
\mathrm{GPa}\end{array}$ \\
\hline Basic honeycomb (Fig. 1) & 0.0 & 0.00 & 2.9 & 11.6 & 4.11 \\
Square-based (Fig. 2) & 1.52 & 6.08 & 7.4 & 29.6 & 0.58 \\
Honeycomb-based (Fig. 6) & 1.40 & 5.60 & 4.8 & 19.2 & 0.62 \\
\hline
\end{tabular}

${ }^{*}$ With the assumption of pure elastic deformation. 

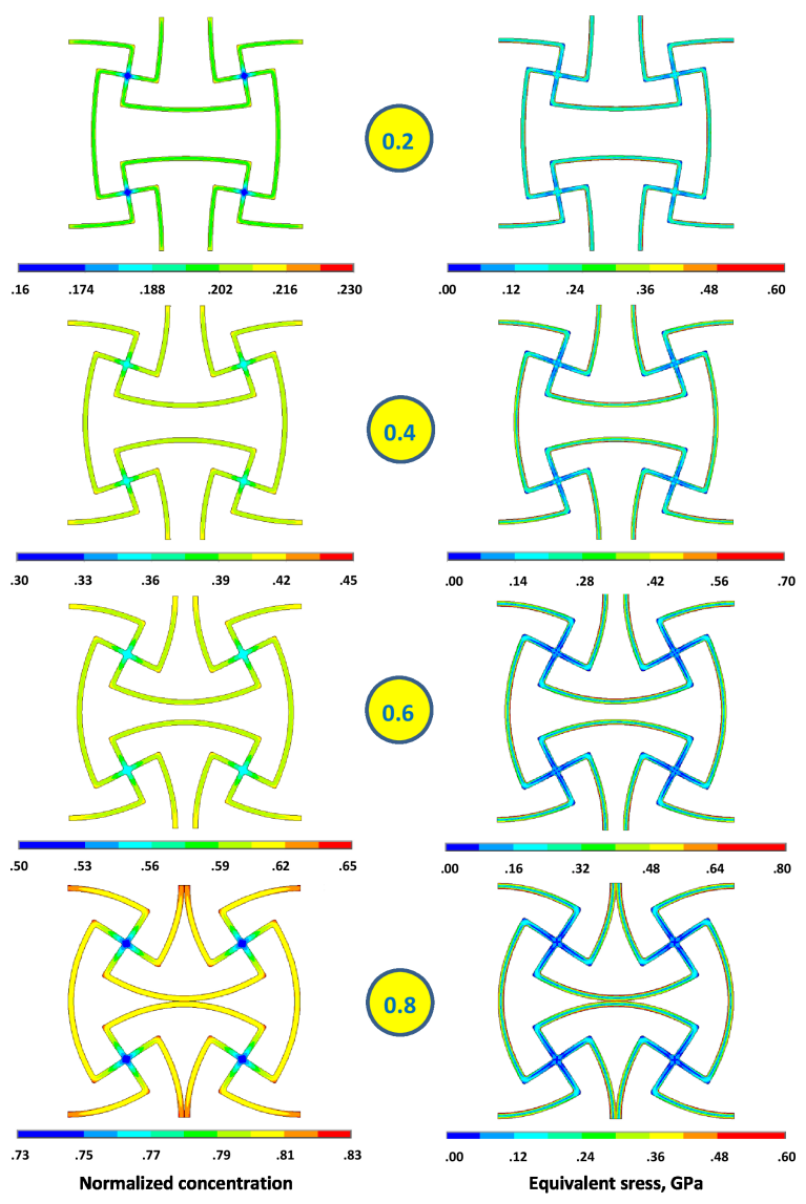

Fig. 3. Geometry and counters of normalized concentration and equivalent stress in optimized square-based microstructure at different stage of lithiation. The average concentration denoted by number in circles.

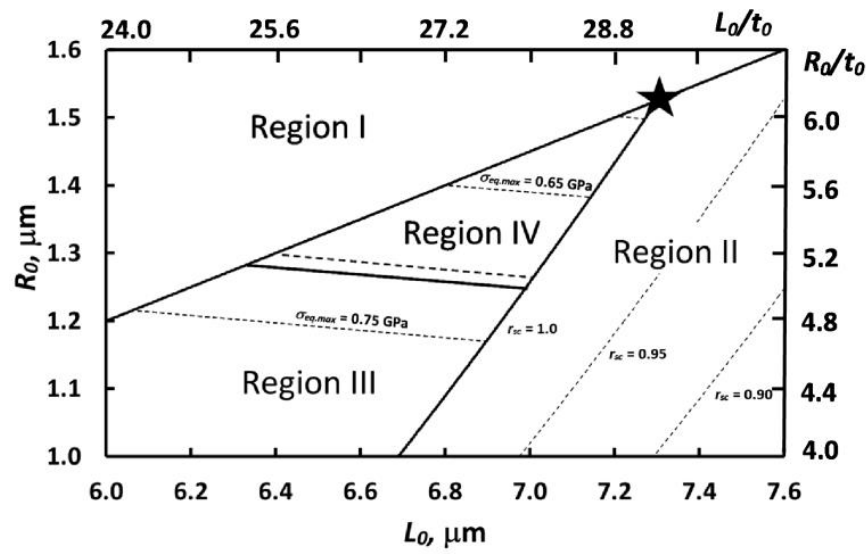

Fig. 4. Optimization of new square-based $\mathrm{Si}$ structure $(80 \%$ lithiation): Region I - overlapping of opposing walls; Region II - low storage capacity; Region III - equivalent stress above yield stress; Region IV - feasible geometry. The optimized geometry is denoted by an asterisk. Isocontours of the maximal equivalent stress and relative storage capacity are denoted by dashed lines.

\subsection{Predefined deformed pattern}

The predefined deformed pattern is another important advantage of the proposed square-based structure (Fig. 5a). In contrast, the basic honeycomb does not provide an unambiguous direction of wall bending (Fig. 5b). During the charge of an anode with multiple cells, the start of bending can fluctuate slightly for different honeycomb walls. The primarily bended structural elements are isolated from each other due to the micro-scale of honeycomb cells and macro-scale of the entire anode. The first bended wall activates the bending of neighboring walls and defines their deformation mode. The bending can occur inwards or outwards of the cell with a probability of $50 \%$. The different patterned arrays grew continuously during charge and, finally, meet each other. The layer between them is severely distorted (Fig. 5b). This results in the fracturing of walls. This drawback is fully rectified with the proposed structure.

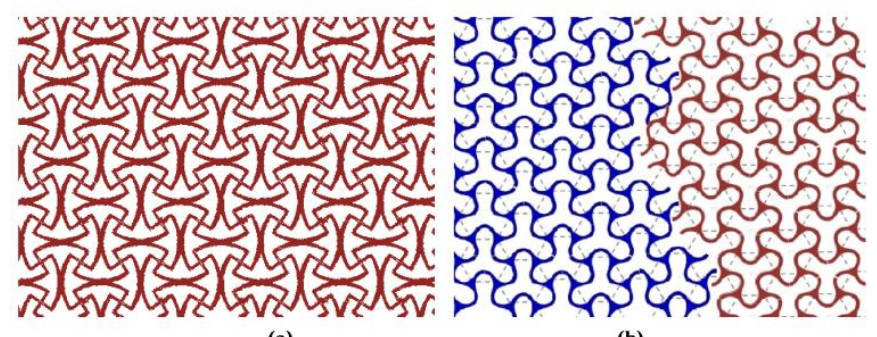

(a)

(b)

Fig. 5. (a) Fully ordered bending in the proposed square-based structure and (b) dissimilar bending patterns in the basic honeycomb structure.

\subsection{Honeycomb-based microstructure}

A similar analysis was performed for the modified honeycomb-based structure presented in Fig. 6. The parameters and the maximal equivalent stress for the optimized honeycomb-based structure are summarized in Table 1. As evident from the table, the new square-based structure results in the lowest equivalent stress after lithiation.

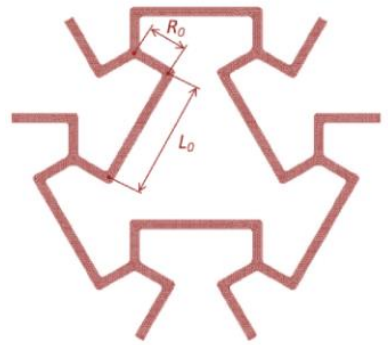

(a)

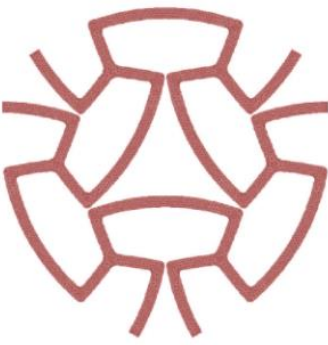

(b)
Fig. 6. Honeycomb-based unit cell: (a) before lithiation, (b) after $80 \%$ lithiation.

\subsection{Verification for entire lithiation cycle}

The effective stress can reach a maximum before lithiation is completed as modeled earlier for the basic honeycomb- and square-patterned anodes [18]. Therefore, an additional verification of the occurrence or absence of plastic deformation throughout the entire charging cycle should be performed. In Fig. 7, the maximal effective stress is compared with the yield stress of lithiated silicon, which is also a function of normalized lithium concentration. The relationship between yield strength of lithiated silicon and $\mathrm{Li}$ concentration is still under investigation. In our study, we assumed a linear dependence $\sigma_{y}(\bar{c})$ 


$$
\sigma_{y}=\sigma_{y 0}-\left(\sigma_{y 0}-\sigma_{y 1}\right) \cdot \bar{c}
$$

where $\sigma_{y 0}=3.6 \mathrm{GPa}$ and $\sigma_{y 1}=0.72 \mathrm{GPa}$ were taken from experiments by Kushima et al. with nanowires [24]. These values correspond to the majority of results obtained by other researchers $[12,25]$. As can be seen in Fig 7, the walls of basic honeycomb start to deform plastically even at early stage of lithiation. In contrast, both new structures (honeycomb- and square-based) remain elastic-deformed throughout the entire lithiation cycle. The developed approach can be applied to the design and analysis of microstructures with geometry different from discussed in this paper.

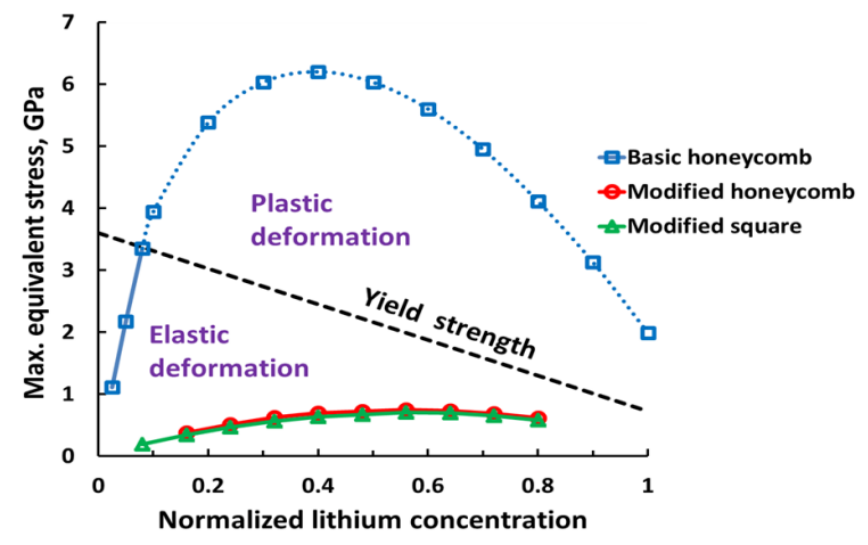

Fig. 7. Development of the maximal equivalent stress during lithiation of different microstructures. The dotted line represents the extrapolation over the yield strength.

\subsection{Application to similar microstructures}

The obtained data are directly applicable to geometrically similar structures. The equivalent stress and footprint-areaspecific storage capacity in such structures remain the same. Therefore the graphs in Fig. 4 and the data in Table 1 can be presented in normalized coordinates $L_{0} / t_{0}-R_{0} / t_{0}$. A change in wall height $h_{0}$ does not influence the bending stress, but leads to a proportional modification in the storage capacity. Thus, the wall height should be as large as technologically possible. If this is the case, the border of Region II in Fig. 4 displaces to the right. As a result, even smaller stress can be achieved with an acceptable storage capacity. Variation in wall thickness leads to a less clear outcome. An enlargement of the wall thickness $t_{0}$ causes a proportional rise in storage capacity, but also a proportional growth in bending stress. Conversely, a reduction in wall thickness results in a proportional diminishing in bending stress, as well as in a proportional decrease in storage capacity. The diagram in Fig. 4 can be easily reconstructed for such cases of study. In addition to patterned silicon anodes, other severely swelling materials and other applications can be studied using the developed approach.

\section{Conclusions}

A new square-based patterned structure for the silicon anode is proposed and optimized. During charge, the optimized structure results in a bending stress below the yield stress of lithiated silicon and pure elastic deformation. This was made possible through the partial replacement of bending deformation induced by $\mathrm{Si}$ swelling with the rotation of structural elements. The optimized structures possess a footprint-area-specific storage capacity that is equal or larger capacity than that of the basic honeycomb-structured Si anode. A similar modification of the honeycomb structure leads to a slightly larger bending stress, but still below the yield stress of fully lithiated silicon. Both new structures maintain perfectly patterned structures over the course of lithiation without strain localization and collapse of structure. The experimental investigation of cycling life of developed silicon anodes is intended in future. The obtained results are directly applicable to geometrically similar structures. The developed approach can be applied to other structures manufactured from severely swelling materials.

The obtained results contributed to the mechanics of metamaterials. For the first time the patterned microstructure under special type of loading, namely the sever swelling, was analyzed and topologically optimized. Developed approach can be applied to other metamaterials with various type of unit cell including 3D structures. As a practical application this could be a 3D-patterned $\mathrm{Si}$ anode maintained all dimensions during operation.

\section{Acknowledgment}

This study was supported by the Institute of Energy and Climate Research - Materials Synthesis and Processing (IEK1), Forschungszentrum Jülich GmbH, Germany

\section{References}

[1] B. Liang, Y. Liu, Y. Xu, Silicon-based materials as high capacity anodes for next generation lithium ion batteries, J. Power Sources 267 (2014) 469-490, doi: 10.1016/j.jpowsour.2014.05.096

[2] M.N. Obrovac, L. Christensen, Structural changes in silicon anodes during lithium insertion/extraction, Electrochem. Solid-State Lett. 7 (2004) A93A96, doi: 10.1149/1.1652421

[3] H. Wu, Y. Cui, Designing nanostructured Si anodes for high energy lithium ion batteries, Nano Today, 7, (2012), 414-429, doi: 10.1016/j.nantod.2012.08.004

[4] S. Goriparti, E. Miele, F. De Angelis, E. Di Fabrizio, R.P. Zaccaria, C. Capiglia, Review on recent progress of nanostructured anode materials for Li-ion batteries, J. Power Sources 257 (2014) 421-443, doi: 10.1016/j.jpowsour.2013.11.103

[5] M. Wang, X. Xiao, Investigation of the chemo-mechanical coupling in lithiation/delithiation of amorphous Si through simulations of Si thin films and Si nanospheres, J. Power Sources 326 (2016) 365-376, doi: 10.1016/j.jpowsour.2016.07.011

[6] D. Wang, Y. Wang, Y. Zou, C. Lu, Z. Ma, Anisotropic mechanical properties of $\mathrm{Si}$ anodes in a lithiation process of lithium-ion batteries, Acta Mech. 229 (2018) 3293-3303, doi: 10.1007/s00707-018-2169-4

[7] H. Yang, F. Fan, W. Liang, X. Guo, T. Zhu, S. Zhang, A chemo-mechanical model of lithiation in silicon, J. Mech. Phys. Solids 70 (2014) 349-361, doi: 10.1016/j.jmps.2014.06.004

[8] M. Pharr, K. Zhao, X. Wang, Z. Suo, J.J. Vlassak, Kinetics of initial lithiation of crystalline silicon electrodes of lithium-ion batteries, Nano Lett. 12 (2012) 5039-5047, doi: 10.1021/n1302841y

[9] Y. Gwak, J. Moon, M. Cho, Multi-scale analysis of an electrochemical model including coupled diffusion, stress, and nonideal solution in a silicon thin film anode, J. Power Sources 307 (2016) 856-865, doi: 10.1016/j.jpowsour.2016.01.037

[10] B. Hertzberg, J. Benson, G. Yushin, Ex-situ depth-sensing indentation measurements of electrochemically produced $\mathrm{Si}-\mathrm{Li}$ alloy films, Electrochem. Commun. 13 (2011), 818-821, doi: 10.1016/j.elecom.2011.05.011

[11] V.B. Shenoy, P. Johari, Y. Qi, Elastic softening of amorphous and crystalline $\mathrm{Li}-\mathrm{Si}$ phases with increasing $\mathrm{Li}$ concentration: A firstprinciples study. J. Power Sources 195 (2010), 6825-6830, doi: 10.1016/j.jpowsour.2010.04.044

[12] V.A. Sethuraman, M.J. Chon, M. Shimshak, V. Srinivasan, P.R. Guduru, In situ measurements of stress evolution in silicon thin films during 
electrochemical lithiation and delithiation, J. Power Sources 195 (2010) 5062-5066, doi: 10.1016/j.jpowsour.2010.02.013

[13] K. Zhao, W.L. Wang, J. Gregoire, M. Pharr, Z. Suo, J.J. Vlassak, E. Kaxiras, Lithium-assisted plastic deformation of silicon electrodes in lithium-ion batteries: A first-principles theoretical study, Nano Lett. 11 (2011) 2962-2967, doi: 10.1021/nl201501s

[14] H. Wang, H.B. Chew, Molecular dynamics simulations of plasticity and cracking in lithiated silicon electrodes, Extreme Mech. Lett. 9 (2016) 503515, doi: 10.1016/j.eml.2016.02.020

[15] L. Baggetto, D. Danilov, P.H.L. Notten, Honeycomb-structured silicon: Remarkable morphological changes induced by electrochemical (de) lithiation, Adv. Mater. 23 (2011) 1536-1566, doi: 10.1002/adma.201003665

[16] X. Yu, J. Zhou, H. Liang, Z. Jiang, L. Wu, Mechanical metamaterials associated with stiffness, rigity and compressibility: A brief review, Progress in Mater. Sci. 94 (2018) 114-173, doi: 10.1016/j.pmatsci.2017.12.003

[17] T. K. Bhandakkar, H.T. Johnson, Diffusion induced stresses in buckling battery electrodes, J. Mech. Phys. Solids 60 (2012) 1103-1121, doi: 10.1016/j.jmps.2012.02.012

[18] A.M. Laptev, Y.C. Malede, S. Duan, R. Mücke, D. Danilov, P.H.L. Notten, O. Guillon, Modeling large patterned deflection during lithiation of microstructured silicon, Extreme Mech. Lett. 15 (2017) 145-150, doi: 10.1016/j.eml.2017.05.001

[19] N. Ding, J. Xu, Y.X. Yao, G. Wegner, X. Fang, C.H. Chen, Determination of the diffusion coefficient of lithium ions in nano-Si, Solid State Ionics 180 (2009) 222-225, doi: 10.1016/j.ssi.2008.12.015

[20] L. Baggetto, R.A.H. Niessen, F. Roozeboom, P.H.L. Notten, High energy density all-solid-state batteries: A challenging concept towards 3D integration, Adv. Funct. Mater. 18 (2008) 1057-1066, doi: 10.1002/adfm.200701245

[21] S. Prussin, Generation and distribution of dislocations by solute diffusion, J. Appl. Phys. 32 (1961) 1876-1881, doi: 10.1063/1.1728256

[22] M. Pharr, Z. Suo, J.J. Vlassak, Variation of stress with charging rate due to strain-rate sensitivity of silicon electrodes of Li-ion batteries, J. Power Sources 270 (2014) 569-575, doi: 10.1016/j.jpowsour.2014.07.153

[23] X. Zhang, W. Shyy, A.M. Sastry, Numerical simulation of intercalationinduced stress in Li-ion battery electrode particles, J. Electrochem. Soc. 154 (2007) A910-A916, doi: 10.1149/1.2759840

[24] A. Kushima, J.Y. Huang, J. Li, Quantitative fracture strength and plasticity measurements of lithiated silicon nanowires by in situ TEM tensile experiments, ACS Nano 6 (2012) 9425-9432, doi: 10.1021/nn3037623

[25] S.T. Boles, C.V. Thompson, O. Kraft, R. Mönig, In situ tensile and creep testing of lithiated silicon nanowires, Appl. Phys. Lett. 103 (2013) 263906, doi: $10.1063 / 1.4858394$ 